Revue

de Sémantique

et Pragmatique
Revue de Sémantique et Pragmatique

38 | 2015

Le futur

\title{
Le futur périphrastique français avec aller : un renvoi spécifique à l'avenir ou un temps en voie de grammaticalisation? Une approche contrastive
}

\section{Aude Rebotier}

\section{(2) OpenEdition}

Journals

Édition électronique

URL : http://journals.openedition.org/rsp/502

DOI : $10.4000 /$ rsp. 502

ISSN : 2610-4377

Éditeur

Presses universitaires d'Orléans

Édition imprimée

Date de publication : 1 septembre 2016

Pagination : 11-34

ISSN : 1285-4093

Référence électronique

Aude Rebotier, « Le futur périphrastique français avec aller : un renvoi spécifique à l'avenir ou un temps en voie de grammaticalisation ? Une approche contrastive », Revue de Sémantique et Pragmatique [En ligne], 38 | 2015, mis en ligne le 01 décembre 2016, consulté le 11 avril 2020. URL http://journals.openedition.org/rsp/502 ; DOI : https://doi.org/10.4000/rsp.502

Ce document a été généré automatiquement le 11 avril 2020

Revue de Sémantique et Pragmatique 


\title{
Le futur périphrastique français avec aller : un renvoi spécifique à l'avenir ou un temps en voie de grammaticalisation? Une approche contrastive
}

\author{
Aude Rebotier
}

1 L'existence en français contemporain de deux formes de futur a fait l'objet de nombreuses études cherchant à définir les spécificités de chacune d'elles. Si l'on admet que les temps verbaux sont issus d'un processus de grammaticalisation, on peut chercher à définir la spécificité du futur périphrastique (FP) par rapport au futur simple (FS) par une différence de degré de grammaticalisation, ou par des chemins de grammaticalisation différents.

2 J'aborderai la question par la comparaison avec le futur simple d'une part, et avec les futurs de deux autres langues d'autre part. L'étude d'un corpus parallèle trilingue permet de classer les occurrences de FS et de FP sans faire appel à des catégories prédéfinies, c'est l'observation des temps correspondants en traduction qui détermine les catégories et dresse " en aveugle " un tableau des emplois des deux temps. Le principe est sensiblement le même que celui utilisé par Dahl (1985) pour identifier les catégories verbales temporelles, aspectuelles et modales de façon translinguistique, à la différence qu'il se basait sur un questionnaire, inévitablement élaboré en fonction des emplois attendus. Un corpus parallèle spontané évite ce biais, mais en introduit un autre : on peut supposer une influence de la langue source sur la traduction. Cependant, ce risque est réduit par le fait que le corpus est constitué à parts égales de textes originaux des trois langues. D'autre part, l'uniformisation induite par la traduction est modérée, puisque les résultats montrent des différences importantes entre les trois langues, dont il est possible de tirer des conclusions. Les deux langues choisies, allemand et italien, sont intéressantes à plusieurs titres pour la comparaison 
avec le français (emploi plus développé du présent futural et des verbes modaux, absence de futur périphrastique).

3 Après une présentation de la problématique (1), l'étude de corpus distinguera emplois non temporels du FP et du FS, c'est-à-dire qui ne réfèrent pas à l'avenir (2), et temporels (3). ${ }^{1}$

\section{PROBLÉMATIQUE}

4 Le futur périphrastique français aller (au présent ou à l'imparfait de l'indicatif) + infinitif (FP) est le résultat d'un processus de grammaticalisation que l'on peut résumer par les étapes suivantes (Vetters \& Lières 2009, 27-28, Vet 1993, 72, Bybee, Pagliuca \& Perkins 1991, 31):

(i)

Association de deux verbes qui gardent chacun leur sens lexical : aller exprime un déplacement dans l'espace.

(1) - Où vas-tu? - Je vais nager.

(ii)

Phase ou aspect (prospectif) : aller prend un sens figuré et désigne la phase préparatoire du procès à venir.

(2)

Je t'aime encore dix mille fois mieux depuis que tu vas avoir cette robe.

(Damourette \& Pichon : 276, 1930)

(3) Laforgue le stoppa au moment où il allait franchir la porte. (Pennac)

5 Un éventuel complément de temps date la phase préparatoire et non le procès luimême. Dans (3), il s'agit du moment où le sujet est sur le point de franchir la porte, et non où il la franchit réellement.

(iii)

Temps : la forme peut être considérée comme un futur à partir du moment où le complément de temps date le procès à l'infinitif :

(4)

j'ai vraiment hate de partir mais en même temps j'ai peur du moment ou je vais vraiment me retrouver toute seule avec un appart et tout a gerer... [sic] (http://forum.doctissimo.fr 2005)

6 La locutrice évoque le moment où elle se retrouvera seule, non sa phase préparatoire. La prédication principale porte donc sur le procès à l'infinitif.

7 Le FS a connu un processus similaire. Dans les deux cas, entre (i) et (iii), un élément passe du statut de lexème (le verbe aller ou avoir) au statut d'auxiliaire (aller) ou de morphème grammatical lié (désinence du futur synthétique), dans un système où il commute non plus avec d'autres lexèmes mais avec d'autres temps. Le processus correspond à la définition de la grammaticalisation par Traugott \& Konig $(1991,189)$ : « a dynamic, unidirectional historical process whereby lexical items in the course of time acquire a new status as grammatical, morpho-syntactic forms, and in the process come to code relations that either were not coded before or were coded differently ». 
i) Verbe plein

ii) Phase

iii) Temps a) justifié

b) non justifié

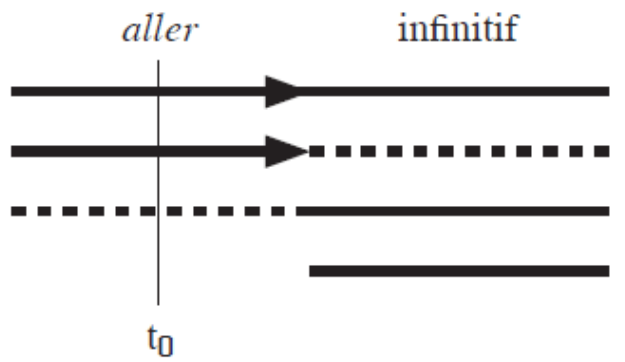
logiquement aboutir au procès futur (exemples 5 à 7 , la glose proposée par les auteurs met en évidence le procès en cours).

\begin{tabular}{|l|l|}
\hline (5) & Pierre va mourir = p.ex. la maladie a commencé son travail de sape (Wilmet 2003,414) \\
\hline (6) & $\begin{array}{l}\text { Paul va se marier = Paul se prépare au mariage, la fiancée existe. (Confais 1995, } 399 ; \\
\text { Damourette et Pichon1911-1936, 278) }\end{array}$ \\
\hline (7) & Marie va avoir un bébé. = le bébé est en route. (Confais 1992, 86) \\
\hline (6a) & Paul se mariera. (Confais 1995, 399) \\
\hline (7a) & Marie aura un bébé = pure conviction (Confais 1992, 86) \\
\hline
\end{tabular}

12 En-dehors de ces emplois types, le lien peut être plus délicat à définir. Wilmet (2003, 413) parle de «processus enclenché» avec les verbes perfectifs (va mourir), alors que la différence entre le FP et le FS serait «d'ordre intellectuel» avec les verbes imperfectifs.

Dans tous les cas, au FP, l'assertion sur un événement à venir serait justifiée par quelque chose (un procès, une condition) existant au moment de l'énonciation et représenté par les pointillés dans le schéma 1 (iiia). Le FS au contraire pourrait situer un événement dans l'avenir sans faire de lien avec le présent, comme dans les exemples (6a) et (7 a) :

« Le FUT (=FS), au contraire [du FP], suggère que le locuteur n'a aucune preuve et ne s'appuie que sur son intuition. » (Confais 1995, 399) 

pas accès aux emplois non justifiés, de type (iiib), comme le laisse entendre la citation de Confais (1995). En effet, le FP, qui date du XV siècle, est plus récent que le FS, apparu en bas-latin. D'autre part, contrairement au FS dont les composantes (infinitif + habeo) ont fusionné, il n'a aucun des indices formels de grammaticalisation : écriture en un mot, existence d'allomorphes, modification du radical du verbe principal, réduction phonologique (Bybee, Pagliuca \& Perkins 1991, 34-37, Bybee, Perkins \& Pagliuca 1994, 6-8). Le lien avec le présent reste matérialisé par la présence de l'auxiliaire aller. ${ }^{2}$

Dahl $(2000,315)$ interprète ainsi l'opposition entre FP et FS comme la concurrence entre une forme ancienne et une forme plus récente, qui n'a pas encore acquis toutes les caractéristiques d'un vrai futur. Pour Vet $(1993,79)$, FP et FS sont largement interchangeables en présence d'un complément de temps, autrement dit, le FP serait totalement grammaticalisé dans certains emplois. ${ }^{3}$

18 b) Le FP et le FS ne suivent pas le même chemin de grammaticalisation. Schrott (1997, 142) refuse ainsi l'idée que le FP soit simplement en train de remplacer le FS, et définit leur coexistence comme un moyen d'enrichir les façons de référer à l'avenir. Elle note toutefois une tendance du FP à évoluer et à se détacher du conditionnement qui, d'après elle, le définit.

19 En particulier, le lien avec le présent, ou, en d'autres termes, la nature de la justification dépend évidemment du type d'auxiliaire utilisé, elle sera différente avec aller et avec des constructions exprimant la volonté (will en anglais), la nécessité, ou la possibilité (sens d'origine du FS). Il se peut que le type d'auxiliaire explique certaines différences entre FP et FS, ces différences étant amenées à s'estomper au fur et à mesure de la grammaticalisation.

20 Pour tenter d'évaluer la part des deux facteurs dans les spécificités du FP, nous procèderons à un tableau de ses emplois en comparaison avec ceux du FS, aussi bien non temporels (2) que temporels (3). 


\section{LES EMPLOIS NON TEMPORELS DU FP}

Les emplois non temporels des futurs, qui recouvrent une grande partie de leurs emplois dits modaux, sont souvent analysés comme un développement ultérieur, une étape iv à ajouter au schéma 1 (Bybee, Pagliuca \& Perkins 2001). ${ }^{4}$

Si le FS et le FP, dans leurs emplois temporels, ne réfèrent pas à l'avenir de la même façon (hypothèse $b$ présentée ci-dessus), il se peut qu'ils développent des emplois non temporels différents. Si en revanche la différence est liée au degré de grammaticalisation, le FP, moins avancé, devrait simplement avoir moins accès aux emplois modaux et plus généralement non temporels. C'est notamment la position de Fleischman (1982), pour qui la modalisation des FS dans les langues romanes a conduit à l'apparition de FP pour les remplacer dans les emplois temporels ; ces FP, plus récents, auraient à peine commencé leur propre modalisation.

Un tableau des emplois va permettre de tester cette hypothèse, en montrant si le FP a ou non des emplois modaux moins développés, ou différents de ceux du FS.

4 Je recense ici uniquement les emplois non temporels d'aller + infinitif, c'est-à-dire ceux où le procès n'est pas situé dans l'avenir. ${ }^{5}$ Ces emplois n'étant pas tous représentés dans le corpus trilingue, il s'agit d'un tableau non quantitatif, basé sur une synthèse de la littérature sur le sujet.

\section{ALLURE EXTRAORDINAIRE}

Aller signale ici «un caractère dérangeant par rapport à l'ordre attendu des choses» (Damourette \& Pichon 1911-1936, 107 \$1652). Dans les emplois types ${ }^{6}$, il peut être supprimé (le verbe à l'infinitif prenant le mode et le temps d' aller) sans autre effet que la perte de cette nuance.

\begin{tabular}{|l|l|}
\hline$(8)$ & Mais va lui expliquer ça, à l'autre, dans son uniforme. $($ Pennac $)=$ Mais explique-lui ça \\
\hline$\left(8^{\prime}\right)$ & Ma vallo a spiegare a quello lî, con la sua uniforme. ${ }^{7}$ \\
\hline
\end{tabular}

Deux faits semblent indiquer que l'extraordinaire ne s'est pas développé à partir du sens temporel et qu'il s'agit plutôt de formes homonymes. Tout d'abord, contrairement à l'auxiliaire du FP, l'auxiliaire aller n'y est pas limité en mode et en temps. Il peut être ainsi à l'impératif dans l'exemple (8). D'autre part, une forme similaire existe en italien, andare a + infinitif, comme on le voit dans la traduction ( $\left.8^{\prime}\right)$, alors que l'italien ne possède pas par ailleurs de futur formé sur andare.

oordinaire est le seul emploi non temporel non partagé par le FS. Si l'on admet que ce n'est pas un emploi du FP, on peut donc dire que le FP n'a pas développé d'emploi non temporel spécifique.

\section{FUTUR NARRATiF / HiSTORIQUE}

8 Le futur narratif réfère à un événement (réel ou fictif) passé par rapport à l'énonciation (réelle ou fictive). L'origine de cet emploi est temporelle : il s'agit d'annoncer un 
événement à venir par rapport à un point de vue passé, que représente le présent narratif. Mais il peut s'inscrire dans une séquence d'événements, et n'est alors plus senti comme une anticipation :

Il épousa Pauline, seconde fille de sainte Paule, mais elle mourra en 397, après seulement trois

(9) ans de mariage. Il fit alors dire un office pour le repos de son âme, et offrit un festin à tous les pauvres de Rome. (Wikipédia, article Pammaque)

'elle mourra en 397' n'est plus une parenthèse anticipatrice, c'est une étape du récit qui a sa place entre 'il épousa Pauline' et 'il fit dire un office'.

Comme le FS, le FP est utilisé comme futur narratif :

(10) De ce voyage va naître un livre (...), qui sera publié en 1834. (Wikipédia, article L’Or de Paris)

31 En étudiant sur corpus les temps qui pouvaient se substituer au passé simple (Rebotier 2010, 2014), j'ai constaté que par rapport aux principaux temps narratifs (passé simple, passé composé, présent narratif), le futur narratif était un emploi marqué, qui se trouvait dans des contextes syntaxiques très particuliers ; il est par exemple corrélé positivement à la postposition du sujet, comme dans l'exemple (10). Or ces contextes sont sensiblement les mêmes pour le FS et le FP.

\section{FUTUR ÉPISTÉMiQUE / CONjECTURAL}

Le futur épistémique commute avec un présent (ou un passé composé s'il s'agit d'un futur antérieur) accompagné d'un modalisateur :

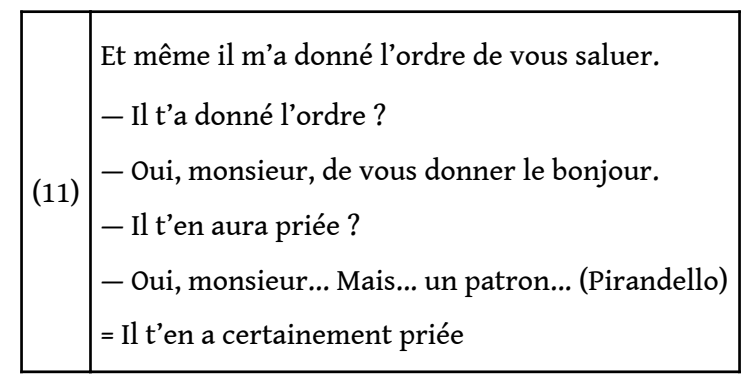

L'origine temporelle de cet emploi est généralement admise : le futur permettrait de repousser dans l'avenir la vérification de l'hypothèse (Damourette et Pichon 1911-1936, 388, Weber 1954, 181, Dahl 1985, 108, Martin 1987, 113, Vetters 1996, 12, Schrott 1997, 294, Dendale 2001, 5) :

« On sonne, ce sera Jean. Il est, d'une part, vrai que le locuteur suppose que Jean est déjà là au moment de la parole, mais l'évaluation de l'hypothèse, d'autre part, est clairement située après le moment de la parole («Vous verrez que c'est Jean »).» (Vetters 1996, 12)

Comme l'emploi narratif, il se détache de la valeur anticipatrice originelle lorsqu'aucune vérification n'est possible. 

différemment (formes synthétiques et différents types d'auxiliaires), comme l'italien et l'allemand dans les correspondants en traduction de l'exemple (11) : explicitement niée par certains (Bündgen 1996, Wilmet 2003, 413, Barcelo 2007, 48), admise par d'autres (Vet 1993, 82). Pour Larreya (2008), le FP épistémique serait même moins rare et moins restreint que le FS, mais on manque d'études de corpus. Il semble en tout cas plus familier, moins littéraire que le FS. On le trouve dans une langue informelle : 
(13) Merde y va avoir oublié l'apéro ! (Larreya 2008, 139)

voila mon probleme j'ai un pokemon de type brave mais je ne sais pas si ce pokemon est de nature brave»2' ou brave «39»

(14)

[Réponse] Si tu l'as déjà fait combattre, c'est un peu la loose, il va avoir pris des EV et tu ne pourras pas vraiment savoir. (pokemongemme.com 2012) = il a certainement pris des EV

Ce serait à confirmer sur corpus, mais il semble que l'emploi conjectural en recul pour le FS soit repris par le FP.

\section{FUTUR GÉNÉRIQUE}

41 Le futur générique qualifie un événement potentiel ou atemporel, généralement itératif, et il commute, selon les cas, avec un présent, ou avec pouvoir au présent.

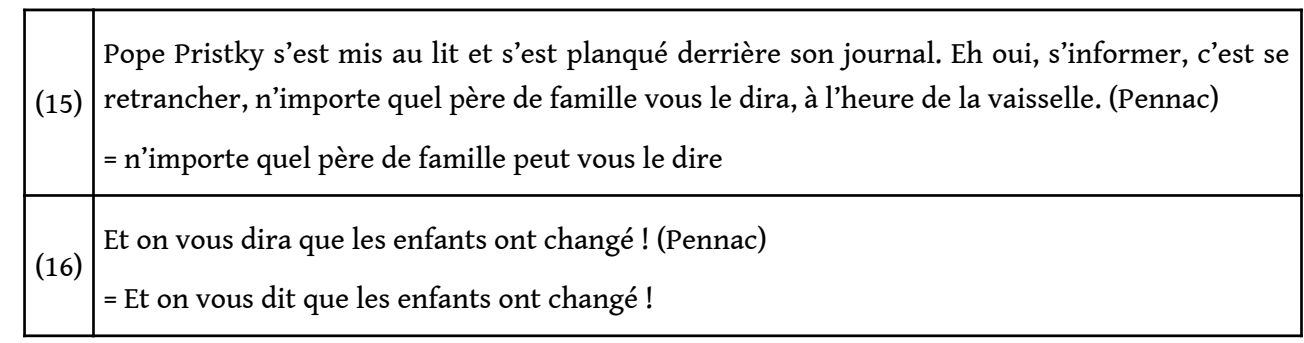

L'emploi, qui existe avec les futurs des trois langues de mon corpus, est certainement dérivé du sens futural ; le futur y exprime l'ultériorité à un autre procès que l'on peut reconstruire :

\begin{tabular}{|l|l|}
\hline (15a) & Demandez à n'importe quel père de famille, il vous le dira \\
\hline (16a) & Et après (ça), on vous dira que les enfants ont changé ! \\
\hline
\end{tabular}

Les exemples de ce type sont tous au FS dans mon corpus. Le FP est toutefois possible, si on explicite l'ultériorité :

\begin{tabular}{|l|l|}
\hline$(16 b)$ & Et après (ça) on va vous dire que les enfants ont changé ! \\
\hline$(16 c)$ & ?? Et on va vous dire que les enfants ont changé ! \\
\hline
\end{tabular}

On trouve d'ailleurs des exemples authentiques de ce type, comme le titre d'un post qui raconte une relation où le garçon se montre instable :

(17) Et après on va dire que les filles sont compliquées !! (2011) 
L'emploi générique semble donc davantage lié à la temporalité pour le FP que pour le FS : il s'agit vraisemblablement d'un emploi émergent pour le FP.

«L'emploi illustratif » décrit par Bres \& Labeau (2013b) et représenté par l'exemple (18) peut être considéré comme un cas particulier de générique ; au caractère potentiel et itératif du procès s'ajoute la particularité d'être un exemple illustratif : " the periphrasis with an illustrative interprétation consists in providing one or several examples illustrating a previous assertion or answering a question related to an activity or a behaviour. That example is shown as iterative and potential. " (Bres \& Labeau 2013b, 188).

(18)

Par moments, il va voir. A d'autres moments, il ne voit plus rien. (Damourette \& Pichon V, 116 $\S 1662)=$ Par moments, il voit

Il n'est pas toujours possible de reconstruire explicitement un procès antérieur dans ces emplois. Cependant, il est admis que le FP y commute sans difficulté avec le FS (Bres \& Labeau 2013b, 189, Damourette \&Pichon V, 117 \$1662, Larreya 2005, 137), ce qui laisse penser qu'il est lié au sens temporel commun aux deux formes.

8 Labeau \& Bres (2013b : 177) considèrent que l'emploi illustratif du FP est en expansion : limité aux conversations à l'origine, il s'étend à différents types de situations (journalistique, scientifique, littéraire), et l'étude d'Abouda et Skovrec (ce volume) confirme que l'essor du FP dans les emplois génériques et illustratifs s'accompagne d'un fort recul du FS. Il y aurait donc ici une spécialisation du FP (dans un rapport concurrentiel avec le FS), mais pas une spécificité (un emploi réservé au FP du fait du sens de son auxiliaire).

\section{ATTÉNUATION / MiTIGATION}

9 Le futur d'atténuation est lui aussi habituellement considéré comme dérivé de l'emploi temporel (Bres \& Labeau 2013a, 21) : il commute avec un présent, mais produit une affirmation moins directe, le futur donnant au destinataire l'illusion de pouvoir s'y opposer. On peut regrouper ici d'une part des verbes de parole employés de façon performative (19) : je vous demanderai, je vous prierai, je me permettrai de, je l'avouerai, je ne vous cacherai pas, je vous répondrai que, j'observerai que, nous dirons, et d'autre part des énoncés exprimant une décision, sans verbe performatif (20).

\begin{tabular}{|l|l|}
\hline$(19)$ & Je vous demanderai de me fournir quelques références, s'il vous plaît. (Brecht) \\
\hline$(20)$ & $\begin{array}{l}\text { Et pour madame, ce sera tout ? - Ce sera tout. (= c'est tout, définitivement tout). (Wilmet 2003, } \\
413)\end{array}$ \\
\hline
\end{tabular}

Le futur d'atténuation est lui aussi souvent réputé inaccessible au FP, lequel pourrait tout au plus exprimer un avenir immédiat, mais pas de performativité (Schrott 1997, 321, Wilmet 2003, 413, Vet 1993, 82). De fait, FS et FP ne sont pas toujours interchangeables. Mais on trouve des emplois similaires au FP, notamment avec des verbes de parole : 
(21) Je vais vous prier de bien vouloir excuser mon indiscrétion (Ionesco)

Premièrement, sainte Jeanne, je vais vous demander de faire que (...) Deuxièmement, sainte

(22) Jeanne, je vous demanderai de faire que (...)

Sixième miracle, je vous demande de nous rendre le respect de l'âge. (V. Volkoff 2005, Prière à Sainte Jeanne d'Arc) ${ }^{8}$

Dans l'exemple (22), l'auteur alterne FP, FS et présent, tous avec la même valeur performative. Le FP peut aussi exprimer une décision. Dans l'exemple (23), la phrase au FP met fin à une liaison, elle est effective dès que la phrase est prononcée, comme le montre la suite du post.

\section{Tu sais quoi on va s'arreter là. [sic]}

(23) C'est ce qu'il m'a dit. (...) Depuis plus de nouvelles, il ne m’a mm pas téléphoné depuis son retour. (http://forum.aufeminin.com 2011)

Tous ces emplois semblent assez formulaires, FS et FP y commutent difficilement. Ainsi, dans (20a) et (23a), les futurs prennent un sens temporel au lieu du sens performatif des originaux (20) et (23).

\begin{tabular}{|l|l|}
\hline$(20 a)$ & Ca va être tout. \\
\hline$(23 a)$ & Tu sais quoi on s'arrêtera là. \\
\hline
\end{tabular}

De même, les expressions avec le verbe dire par lesquelles le locuteur commente le choix des mots qu'il utilise semblent figées : le FP se trouve plutôt avec la première personne du singulier, le FS avec on :

\begin{tabular}{|l|l|}
\hline (24) & $\begin{array}{l}\text { Que n'écrirait-on si ces pressions récurrentes (...) avaient lieu dans des pays, on va dire, plus } \\
\text { exotiques. (Lansari 2010, 280) }\end{array}$ \\
\hline (25) & des mots qui ont un sens, je dirai ‘voisin' à défaut d'un mot plus précis. (Schrott 1997, 331) \\
\hline
\end{tabular}

Mais il est clair que le FP, comme le FS, a accès aux différents types d'emploi d'atténuation.

55 En conclusion, le FP n'a aucun emploi non temporel qui lui serait spécifique ; en revanche, il a développé ou est en train de développer tous les emplois non temporels que connaît le FS. Ceci nous oriente vers l'hypothèse d'un FP en train d'achever sa grammaticalisation, en train de conquérir tous les emplois du futur. 


\section{LES EMPLOIS TEMPORELS DU FP}

De nombreuses études ont décrit dans le détail la concurrence du FP et du FS, sur la base de leur commutation. Le corpus parallèle permet d'aborder la question d'une manière synoptique, en classant les occurrences sur un continuum au moyen d'un modèle multilingue.

\section{RENVOi DiRECT ET iNDiRECT}

Pour référer à l'avenir, le français peut utiliser le FS, le FP ou le présent. Je pose, avec Schrott (1997), que le présent futural signale qu'il existe un plan au moment de l'énonciation qui prévoit la réalisation d'un événement à venir (cf. l'argumentation dans Rebotier 2005).

(26) Mon train part à $10 \mathrm{~h}$.

(26) signifie que le train est indiqué pour 10h sur le planning, et non qu'il partira effectivement à 10h. Il s'agit donc, comme pour les emplois de phase du FP, d'un renvoi indirect à l'avenir. Ce type d'énoncé est régulièrement au présent dans différentes langues (Dahl 2000, 311).

Dans une étude précédente (Rebotier 2009 et 2010), j’ai comparé la concurrence entre futur et présent futural dans trois langues (français, allemand, italien) dans un corpus littéraire parallèle (voir les références en fin d'article). ${ }^{9}$

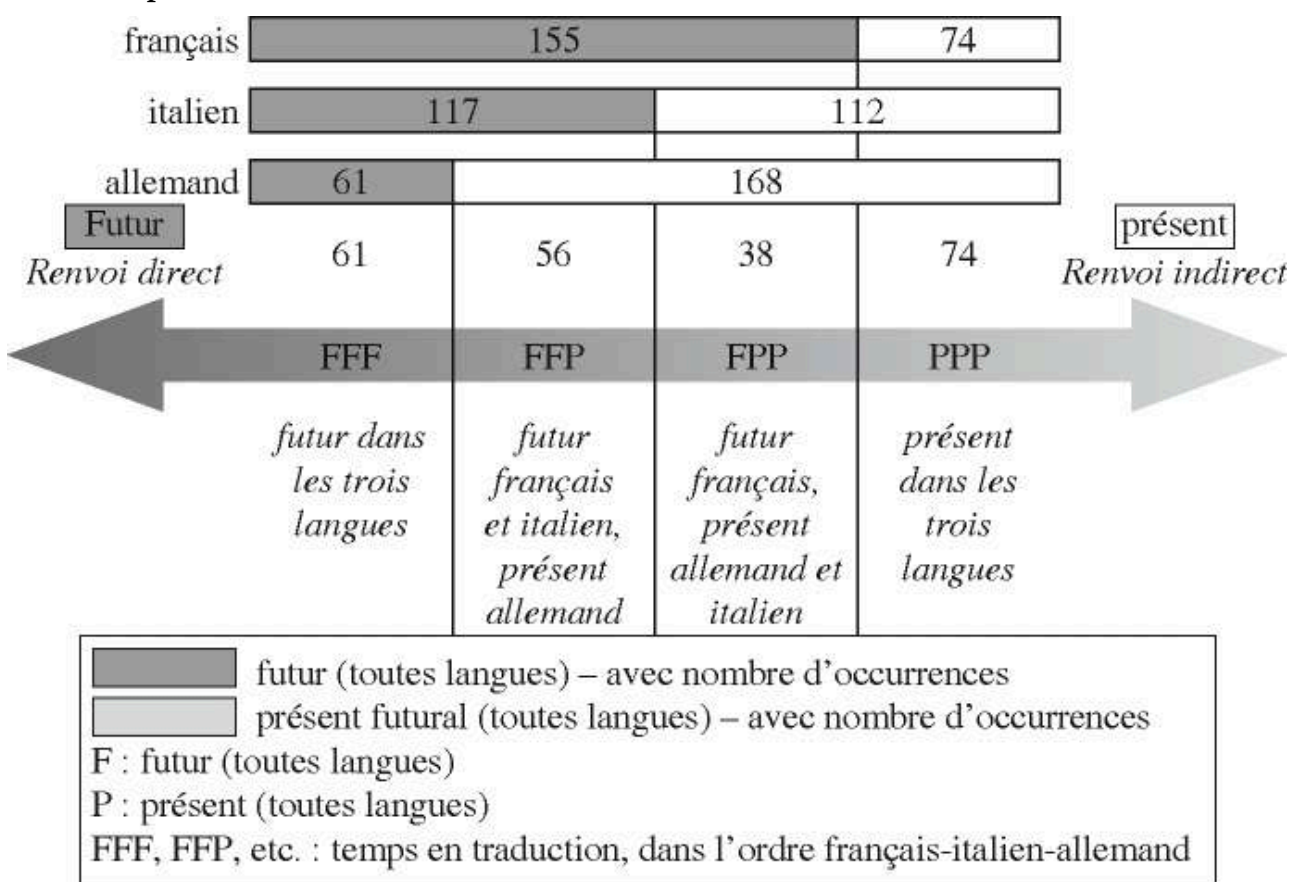

Schéma 2. Traduction des temps futuraux dans un corpus parallèle

Les chiffres (schéma 2) montrent que le pôle du futur et celui du présent coïncident dans les trois langues : parmi les combinaisons théoriquement possibles en traduction, quatre (celles qui apparaissent sur le schéma) sont massivement représentées, les 
quatre autres sont marginales ${ }^{10}$. Un futur allemand est quasiment systématiquement traduit par un futur dans les deux autres langues, un futur italien est régulièrement traduit en français par un futur, mais en allemand soit par un futur soit par un présent.

Cette régularité qui privilégie massivement quatre combinaisons de traduction permet, avant même d'examiner les emplois, de supposer l'existence de catégories translinguistiques (au-delà d'une langue particulière) de futur et de présent futural. Les pôles sont les mêmes, mais l'extension autour de ces pôles varie selon les langues. En français, le présent n'a pas vraiment dépassé le stade du renvoi indirect. L'extension du présent futural est plus large en italien et plus encore en allemand : dans ces deux langues, il est beaucoup moins contraint et il est clairement capable de renvoi direct à l'avenir. En revanche, parmi les futurs français, on peut distinguer un noyau (les énoncés FFF, au futur dans les trois langues) et des emplois marginaux (FPP).

Il faut préciser que le futur allemand, bien que morphologiquement périphrastique (werden + infinitif), ne permet pas la focalisation sur l'auxiliaire, autrement dit, il n'a pas d'emploi de phase. A part sa fréquence modérée, il a toutes les caractéristiques d'un futur grammaticalisé (Rebotier 2006 et 2009).

\section{LE FP EST PLUS PRÈS DU PÔLE iNDiRECT QUE LE FS}

Dans le schéma 2, FP et FS sont considérés tous deux comme des futurs. Si on les distingue, on constate que le FP est plus souvent traduit par des présents que le FS : $66,7 \%$ des FP sont traduits par des présents allemands contre $58,2 \%$ des FS, et $41 \%$ des FP sont traduits en italien par un présent contre $20,2 \%$ des FS. $37,2 \%$ des FP sont traduits par un présent dans les deux autres langues à la fois, contre seulement $17,9 \%$ des FS.

En conséquence, la proportion de FP augmente quand on se rapproche du pôle du présent futural : il représente entre $18 \%$ et $19 \%$ des futurs français dans les emplois proches du noyau du futur (FFF et FFP), 34,5\% dans la catégorie la plus marginale, FPP (schéma 3), la moyenne du corpus étant à $24,7 \%$.

Cette affinité du FP avec le présent futural n'est pas surprenante : contrairement au FS, FP et présent peuvent tous deux exprimer un renvoi indirect à l'avenir, via une assertion sur le présent, même si la nature de l'objet présent est différente : un plan avec le présent futural, une phase préparatoire avec le FP. Vet $(1993,75)$ affirme ainsi que la présence d'un antécédent au moment de l'énonciation oblige à utiliser présent ou FP (l'antécédent étant un ensemble de conditions suffisantes avec le présent, et non suffisantes avec le FP). D'après Schrott $(1997,177)$, le présent futural français commute mieux avec le FP qu'avec le FS, à cause du lien qu'ils ont tous deux avec le moment de l'énonciation. On voit ici que cette affinité existe aussi en traduction, ce qui place le FP dans une position intermédiaire entre le FS (qui a une affinité avec le pôle futur) et le présent futural. 


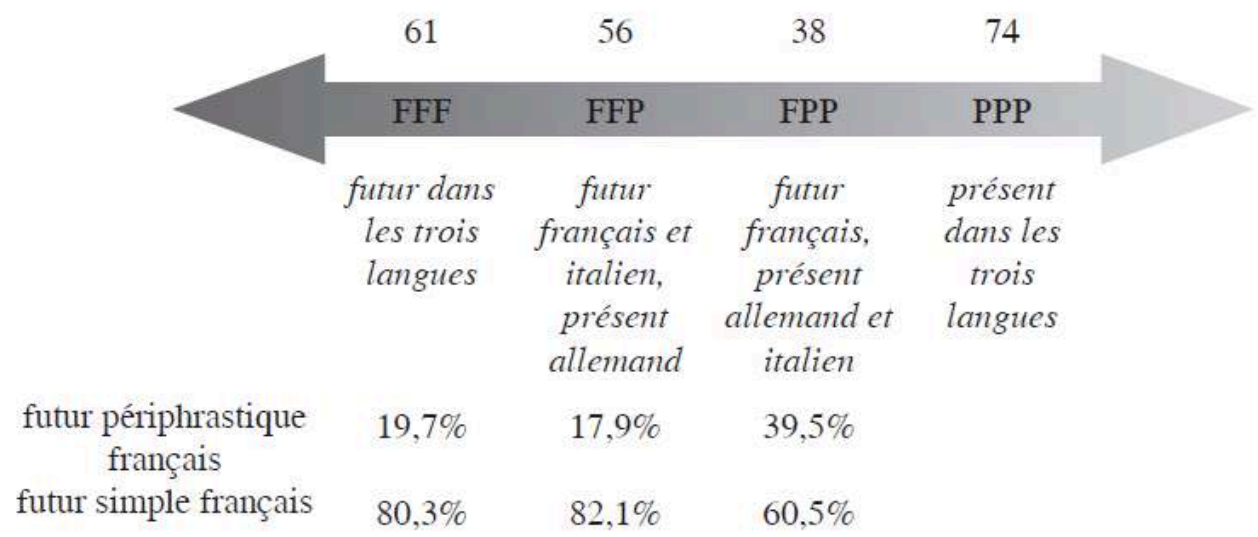
l'opposition futur / présent futural de manière translinguistique : la part des emplois
indépendants de la volonté décroît régulièrement du pôle futur au pôle présent (schéma 5).

\begin{tabular}{|l|l|l|l|}
\hline & FFF & FFP & FPP \\
\hline procès dépendants de la volonté & $32,8 \%$ & $53,5 \%$ & $67,5 \%$ \\
\hline procès indépendants de la volonté & $67,2 \%$ & $46,5 \%$ & $32,5 \%$ \\
\hline
\end{tabular}
traduction trilingue des temps futuraux

La volonté est évidemment en relation avec la notion de plan, propre au présent futural. En revanche, elle ne fait pas directement partie du sémantisme d'aller. D'après Bybee, Perkins et Pagliuca (1994, 5) et Bybee, Pagliuca et Perkins (1991, 31), l'expression de l'intention est néanmoins un stade de développement (entre le sens lexical et le véritable futur) aussi bien pour les futurs formés à l'aide de verbes exprimant le désir 
ou la volonté (comme will en anglais), que pour les futurs formés, comme le FP, sur des verbes de mouvement, le mouvement orienté vers un but étant interprété dans un premier stade d'abstraction comme une intention. Vetters \& Lières $(2009,28)$ situent cette étape pour le FP français au tout début de l'apparition de la périphrase, à partir du XIV ${ }^{\mathrm{e}}$ siècle. Toutefois, parmi les emplois propres au FP, où le lien avec le présent est évident (prospectif ou avenir justifié), certains n'ont rien à voir avec l'intention (voir les cas de mise en garde ci-dessous, exemples 30 à 32). La corrélation entre FP et procès dépendants de la volonté suggère toutefois que l'intention est une interprétation privilégiée de la phase préparatoire du FP. Manifestement, ce critère est commun aux deux types de renvoi indirect à l'avenir représentés ici, le présent futural et le FP.

Les chiffres présentés ici ne prennent pas en compte l'ajout éventuel de périphrases spécifiques. Les emplois de prospectif du FP peuvent se rendre par stare per en italien, im Begriff sein en allemand (être sur le point de). D'autre part, on sait que l'allemand et l'italien utilisent davantage de verbes modaux que le français ; en particulier, ceux qui expriment une volonté peuvent s'employer dans un sens affaibli pour renvoyer à l'avenir : volere (vouloir) en italien ; wollen (vouloir) et sollen (devoir, obligation dérivant de la volonté d'un tiers) en allemand. Ils semblent tout indiqués pour exprimer soit la phase préparatoire, soit l'avenir justifié. Ainsi, Weber $(1954,203)$ estimait que les verbes modaux étaient la moins mauvaise solution pour rendre le sens du FP en allemand. Mon corpus comporte quelques traductions de ce type :

\begin{tabular}{|l|l|}
\hline$(27)$ & $\begin{array}{l}\text { So will ich es euch denn gestehen: ich verkaufe mich, um leben zu konnen (Brecht) (vouloir, } \\
\text { présent) }\end{array}$ \\
\hline$\left(27^{\prime}\right)$ & Eh bien, je vais tout vous dire : je me vends pour vivre [...] \\
\hline$\left(27^{\prime \prime}\right)$ & Ecco: voglio confessarmi a voi: io mi vendo per vivere (vouloir, présent) \\
\hline$(28)$ & Von was soll ich leben? (Brecht) (devoir, présent) \\
\hline$\left(28^{\prime}\right)$ & De quoi allons-nous vivre? \\
\hline$\left(28^{\prime \prime}\right)$ & Di che cosa devo vivere? (devoir, présent) \\
\hline
\end{tabular}

Un verbe modal au présent peut également correspondre à un FS :

\begin{tabular}{|l|l|}
\hline$(29)$ & Ich will euch gern Obdach geben. (Brecht) (vouloir, présent) \\
\hline$\left(29^{\prime}\right)$ & C'est avec plaisir que je vous hébergerai. \\
\hline
\end{tabular}

Le FP est certes plus fréquemment que le FS le correspondant d'un verbe modal en traduction, mais cela reste très minoritaire à côté de la traduction par un présent ou un futur : sur 129 énoncés futuraux dans les trois langues qui sont au FS dans la version française, 5 traduisent ou sont traduits par wollen ou sollen, et un par volere ; sur 41 énoncés futuraux au FP, 6 correspondent à wollen ou sollen, et 6 à volere ou dovere. Encore plus rare est la traduction de la phase par une formule spécifique, comme stare per en italien (être sur le point de - une seule occurrence, pour traduire allait + 
infinitif). Silletti (dans ce volume) fait la même constatation sur son corpus francoitalien journalistique. ${ }^{12}$

\section{LES EMPLOIS SPÉCIfIQUES DU FP}

Si l'on examine les emplois propres à chacun des deux futurs, on constate que ceux du FP sont plutôt situés près du pôle indirect. Ainsi, le FS est difficilement acceptable lorsque le locuteur met en garde, prédisant ce qui va se passer si l'on continue ainsi (si tu ne rentres pas 30, si vous continuez à vous fâcher 31, si vous bougez 32) :

\begin{tabular}{|l|l|}
\hline$(30)$ & Laisse tomber, tu vas prendre froid. (F\&L) \\
\hline$(31)$ & Ne vous fâchez pas comme ça ! Vous allez renverser tout le riz.(Brecht) \\
\hline$(32)$ & $\begin{array}{l}\text { [Le locuteur est en train d'ajuster une robe avec des épingles] Ne bougez pas comme ça, } \\
\text { maman Stilman, ou je vais vous piquer le cul. (Pennac) }\end{array}$ \\
\hline
\end{tabular}

Les énoncés de ce type rentrent dans la catégorie bien identifiée des FP où un procès présent est en cours. Ils sont régulièrement traduits par des présents en allemand ${ }^{13}$, et très souvent (mais pas toujours, cf.32') aussi en italien.

\begin{tabular}{|l|l|}
\hline$\left(30^{\prime}\right)$ & Lascia stare, che prendi solo freddo. (présent) \\
\hline$\left(30^{\prime \prime}\right)$ & LaB es doch sein, du erkaltest dich nur. (présent) \\
\hline$\left(31^{\prime}\right)$ & Non si arrabbi! Sta versando tutto il riso! (présent progressif) \\
\hline$\left(31^{\prime \prime}\right)$ & Seien Sie nicht so zornig! Sie schütten noch den Reis aus! (présent) \\
\hline$\left(32^{\prime}\right)$ & Non si muova cosî, mamma Stilman, o finirô per pungerle il sedere. (futur) (32”) \\
\hline$(32 ”)$ & $\begin{array}{l}\text { Bewegen Sie sich doch nicht andauernd, Mama Stilman, sonst pieks ich Ihnen noch in den } \\
\text { Allerwertesten. (présent) }\end{array}$ \\
\hline
\end{tabular}

Le FP est également le seul temps possible pour l'extraordinaire concernant un procès à venir. Le locuteur s'étonne du comportement annoncé ou prévisible de quelqu'un :

\begin{tabular}{|l|l|}
\hline$(33)$ & Ils ne vont quand même pas se défiler maintenant ? (F\&L) \\
\hline$\left(33^{\prime}\right)$ & Non vorrano mica ritirarsi indietro, adesso? (vouloir, futur) \\
\hline$\left(33^{\prime \prime}\right)$ & Sie wollen sich doch wohl jetzt nicht zurückziehen? (vouloir, présent) \\
\hline
\end{tabular}

Damourette \& Pichon (1911-1936, 110 \$1655), après avoir posé comme principe que l'extraordinaire ne comportait aucune composante temporelle, admettent des emplois mixtes, où aller est à la fois auxiliaire de futur et d'extraordinaire. La traduction italienne avec andare est alors impossible, ce qui laisse penser que dans ce cas aller est 
avant tout auxiliaire de futur. Je compte cet emploi parmi les emplois temporels du FP. Schrott $(1997,261)$, qui ne traite que des emplois futuraux, interprète également l'extraordinaire (futural) à partir de la sémantique du FP.

Ces énoncés sont traduits typiquement dans mon corpus par l'ajout du verbe vouloir, au futur en italien, au présent en allemand, comme dans l'exemple (33). J'ai aussi un cas de traduction allemande par un présent sans ajout de verbe modal. Ils sont donc formellement dans la zone médiane (FFP), mais le futur italien est ici très vraisemblablement épistémique et non temporel, il n'indique donc pas un renvoi direct à l'avenir, mais modalise une assertion sur la volonté présente du sujet grammatical. La présence du modalisateur épistémique wohl dans la version allemande (33") soutient cette interprétation ; l'utilisation du futur (épistémique) avec wollen (vouloir) est d'ailleurs également possible en allemand pour traduire l'extraordinaire futural :

\begin{tabular}{|l|l|}
\hline$(34)$ & Allons, allons ! C'est fini ? Tu ne vas pas encore nous parler de ça! (Troyat) \\
\hline$(34 ')$ & Du wirst doch nicht noch einmal davon anfangen wollen! (Schrott 1997, 262) \\
\hline
\end{tabular}

81 Ces énoncés avec futur épistémique en italien peuvent d'ailleurs expliquer la proportion relativement élevée de FP dans la catégorie FFP (schéma 3).

Tous ces emplois propres au FP sont clairement liés à une constatation au moment de l'énonciation, comme les ajouts réalisés en traduction le suggèrent : outre le verbe modal pour l'intention (33' et 33"), le verbe finire qui indique la fin d'un processus en cours (32'), et même une forme progressive sta versando indiquant que le procès a déjà commencé $\left(31^{\prime}\right)^{14}$. On peut donc les considérer comme des emplois peu grammaticalisés, et il n'est pas surprenant de les trouver traduits soit par des présents, soit par des futurs épistémiques, donc près du pôle de renvoi indirect à l'avenir.

\section{LES EMPLOIS SPÉCIfIQUES DU FS}

Le FP est impossible avec certains compléments temporels : toujours, jamais, et des compléments exprimant que l'on repousse le procès à une date non précisée (plus tard, par la suite, un jour). Vet $(1993,78)$ pense trouver de rares exemples de FP compatibles avec jamais :

(35) Ca ne va jamais bouillir.

Toutefois, jamais n'est pas ici opérateur temporel mais modal, signifiant en aucun cas, car il est compatible avec des indications temporelles (cf. Rebotier 2014).

Les énoncés comportant l'un de ces compléments sont tous au futur en italien, et très souvent aussi en allemand. Ils sont donc dans la partie FFF, parfois FFP, et peuvent être considérés comme des emplois typiques d'un futur, encore inaccessibles au FP.

(36) $\begin{aligned} & \text { Transfusé, mort et enterré ! On ne le verra plus jamais se gratter le cul dans son pyjama! } \\ & \text { (Pennac) }\end{aligned}$ 


\begin{tabular}{|l|l|}
\hline$\left(36^{\prime}\right)$ & Trasfuso, morto e sepolto ! Non lo vedremo mai più grattarsi il culo in pigiama! (futur) \\
\hline$\left(36^{\prime \prime}\right)$ & $\begin{array}{l}\text { Tot und beerdigt! Und wir werden nie wieder sehen, wie er sich im Schlafanzug am Arsch } \\
\text { kratzt! (futur) }\end{array}$ \\
\hline$(37)$ & Désamorçons, désamorçons, on avisera plus tard. (Pennac) \\
\hline$\left(37^{\prime}\right)$ & Smorziamo, smorziamo, rifletteremo dopo. (futur) \\
\hline$\left(37^{\prime \prime}\right)$ & $\begin{array}{l}\text { Entscharfen, ersteinmal entscharfen, spater konnen wir uns etwas einfallen lassen. (pouvoir, } \\
\text { présent) }\end{array}$ \\
\hline
\end{tabular}

L'opposition entre FP et FS semble donc bien se refléter dans le continuum établi sur la base de la seule traduction pour l'expression de l'avenir. Le FS a une affinité avec le noyau du pôle du futur, tandis que le FP est mieux représenté dans les catégories marginales, aussi bien si l'on considère les chiffres (schémas 3 à 5) que les emplois spécifiques à chaque futur. Si l'on admet que le pôle FFF rassemble les emplois les plus typiques d'un temps futur, on voit qu'ils sont occupés essentiellement par le FS et que le $\mathrm{FP}$ le concurrence davantage dans les emplois marginaux, ceux où les langues qui ont un futur moins étendu passent facilement au présent futural. Ces observations, synthétisées dans le schéma 6, vont dans le sens d'un FP en voie de grammaticalisation.

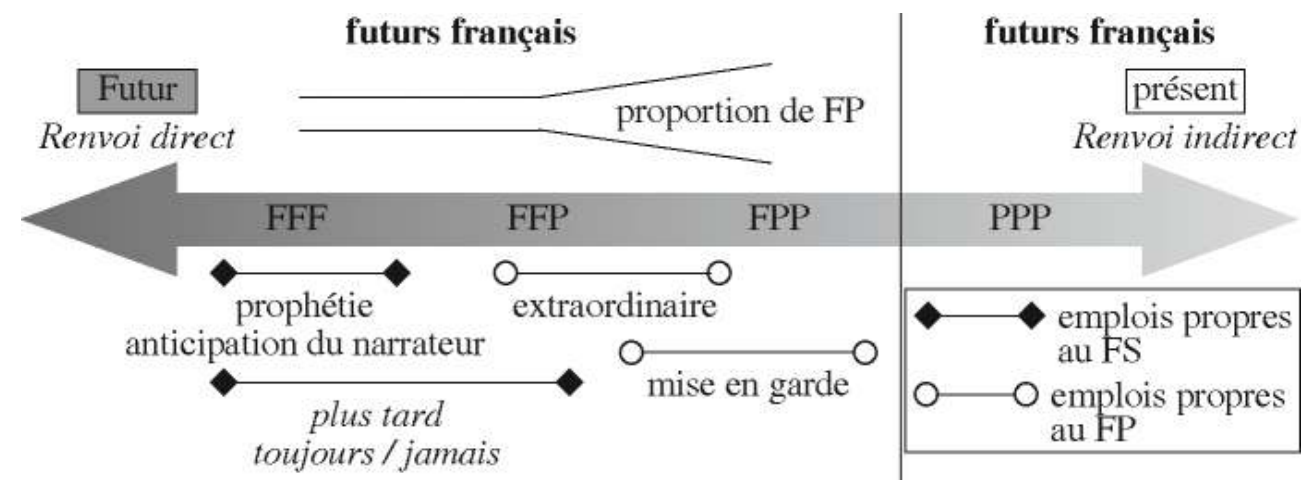

Schéma 6. La traduction du FS et du FP dans leurs emplois futuraux spécifiques

\section{LE FP A ACCÈs AU PôLE FUTUR (FFF)}

Toutefois, le FP n'est pas absent de la catégorie FFF, où il représente $19,7 \%$ des futurs (contre $24,7 \%$ dans l'ensemble des futurs français du corpus). Les prophéties (exemples $38,39,40$ ), ainsi que les anticipations du narrateur omniscient (41) sont au futur dans les trois langues (FFF). Ce sont des emplois typiquement non justifiés, sans lien avec le présent : le locuteur affirme connaître l'avenir, sans autre justification que sa croyance (pour 40 par exemple, croyance en la justice divine).

\begin{tabular}{|l|l|}
\hline (38) & $\begin{array}{l}\text { Ô fille de mon père, le fils du fils de ton fils sera toujours de la deuxième génération, et ses fils } \\
\text { après lui, et les petits qu' engendreront leurs filles, seront de la deuxième génération, des } \\
\text { deux côtés de la mer, pour toujours (Pennac) }\end{array}$ \\
\hline$(39)$ & Tu finiras comme l'oncle Nichi. (F\&L) \\
\hline
\end{tabular}


Canaille, faux-jeton! Ah, tu n'as pas de religion ! Eh bien, vous serez punis de votre

(40) indifférence, vous mijoterez tous dans la poix bouillante! Les dieux vous crachent dessus! Mais vous vous en repentirez! Vous le paierez cher! Vous tous, et les enfants de vos enfants jusqu'à la quatrième génération! (Brecht)

les portières claquent, la vie reprend son cours, emportant sur ses sièges de cuir fin un

(41) héritier on ne peut plus dynamique qu'une rupture d'anévrisme anéantira douze ans plus tard, au cours d'un conseil d'administration parfaitement maîtrisé, au demeurant.

Or, si le FS est très majoritaire dans ces emplois de prédiction pure, le FP n'y est pas impossible. J'ai relevé un cas d'anticipation du narrateur au FP :

Une existence entière. Qui va basculer aujourd'hui, à seize heures et vingt-cinq minutes, à

(42) cause de trois petits cons que rien ne distingue jusqu'à présent des vingt-sept autres, (...) (Pennac)

On peut donc conclure que le FP, majoritairement situé dans la zone marginale du futur, a un accès, limité mais attesté, à certains des emplois les plus typiques du pôle futur. Il répond bien aux caractéristiques d'un temps en voie de grammaticalisation.

\section{LE CRITÈRE DE L'ÉLOIGNEMENT}

91 Malgré son appellation traditionnelle de futur proche, le FP n'est évidemment pas limité à l'expression de l'avenir proche (Damourette et Pichon 1911, 1936, 283). Plus généralement, les verbes signifiant aller, contrairement à ceux qui signifient venir, ne donnent pas lieu à des futurs de proximité (Bybee, Pagliuca et Perkin 1991, 32). Néanmoins, l'éloignement temporel entre l'énonciation et le procès est un critère non négligeable pour la concurrence entre FP et FS (Dahl 2000, 315).

En effet, le FS a perdu la possibilité de référer à l'avenir immédiat, qu'il avait encore en ancien français :

Tu ne sés que te dirai / Compains ? je me marierai. (Damourette et Pichon, 1911-1936, 289,

(43) $\$ 1771)$

$=\mathrm{Tu}$ ne sais pas ce que je vais te dire, compagnon ? Je vais me marier (paraphrase de Damourette et Pichon)

93 A cette époque, le FS n'était pas encore concurrencé par le FP. En italien, où il n'a pas eu à subir cette concurrence, il a toujours ce type d'emplois (44'), et c'est également possible en allemand, qui a pourtant le moins fréquemment recours au futur (45': l'explication suit immédiatement).

(44) Ich wende mich sofort an Herrn Tscheng. (Brecht) (présent) 


\begin{tabular}{|l|l|}
\hline$\left(44^{\prime}\right)$ & $\begin{array}{l}\text { Mi rivolgerô subito al signor Ceng. (futur) } \\
=\text { je m'adresse / je vais m'adresser tout de suite à M. Tcheng }\end{array}$ \\
\hline$\left(44^{\prime \prime}\right)$ & *Je m'adresserai tout de suite à M. Tcheng \\
\hline$(45)$ & Je vais essayer d'expliquer. (Parlement européen, 2001, original en anglais) \\
\hline$\left(45^{\prime}\right)$ & Ich werde versuchen, Ihnen die Gründe dafür zu erlautern. (futur) \\
\hline$\left(45^{\prime \prime}\right)$ & Cercherô di spiegarmi. (futur) \\
\hline$(45 a)$ & ?? J'essayerai d'expliquer. \\
\hline
\end{tabular}

\section{CONCLUSION}

\section{Il s'agit donc d'emplois que le FP a pris au FS.}

L'éloignement temporel est un critère pertinent pour l'ensemble des emplois où les deux futurs sont en concurrence. On peut supposer que les événements proches sont plus facilement mis en relation avec le présent, et que si le FS est plus apte que le FP à exprimer un futur non justifié, il est logiquement plus souvent associé à des événements lointains. Cependant, le critère de l'éloignement joue aussi en l'absence de lien avec le présent. Ainsi, le FP est utilisé en prédiction pure pour des procès relativement prochains (ex.42). Il serait plus étrange pour une annonce lointaine :

? la vie reprend son cours, emportant sur ses sièges de cuir fin un héritier on ne peut plus

(41a) dynamique qu'une rupture d'anévrisme va anéantir douze ans plus tard, au cours d'un conseil d'administration parfaitement maîtrisé

Tout comme le FS serait surprenant pour une prophétie très proche :

(42a) ? Une existence entière. Qui basculera aujourd'hui, à seize heures et vingt- cinq minutes

Si l'on considère l'ensemble de leurs emplois, la concurrence entre les futurs du français a bien des caractéristiques d'une concurrence entre un temps grammaticalisé, le FS, face à un FP qui n'a pas achevé sa grammaticalisation.

En ce qui concerne le développement des emplois futuraux, le FP est moins avancé que le FS. Contrairement à lui, il peut toujours exprimer la phase, stade antérieur au véritable emploi temporel. Selon le critère de la traduction, il apparaît comme un intermédiaire entre le renvoi indirect à l'avenir (représenté par le présent futural) et le renvoi direct à l'avenir, sans justification. Mais par ailleurs, le FP est capable d'assertions non justifiées sur l'avenir, il est apte notamment aux emplois de prédiction 
; et plus généralement, il est moins fréquent, mais tout de même représenté dans la catégorie typique où les trois langues étudiées choisissent d'employer un futur.

Le FP a en outre accès aux emplois modaux qui se développent à partir du sens futural, c'est-à-dire à un stade ultérieur de grammaticalisation. Dans ce stade, il est moins présent que le FS et certains de ses emplois semblent encore émergents, mais il n'est absent d'aucun type d'emploi modal que connaît le FS. D'autre part, si l'on admet que l'allure extraordinaire n'est pas issue de l'emploi futural, le FP n'a pas développé d'emploi non temporel propre qui reflèterait sa spécificité. Les deux futurs semblent donc suivre tous les deux le même chemin, avec un décalage chronologique.

Cependant, le critère de l'éloignement temporel semble traverser l'ensemble des emplois temporels, justifié ou non, c'est-à-dire indépendamment du degré de grammaticalisation. La situation de concurrence actuelle entre les deux futurs semble donc se caractériser aussi par une spécialisation des futurs, le FS étant évité pour les procès proches du moment de l'énonciation.

\section{BIBLIOGRAPHIE}

Barcelo, G.J. (2007), « Le(s) futur(s) dans les langues romanes : évolution linéaire ou cyclique ? ", in Labeau, E., Vetters, C. et Caudal, P. (éd.), Sémantique et diachronie du système verbal français (Cahiers Chronos 16), Amsterdam, New York : Rodopi, 47-62.

Bres, J. et Labeau, E. (2013a), « Aller et venir : des verbes de déplacement aux auxiliaires aspectuels-temporels-modaux », Langue française 179, 13-28.

Bres, J. et Labeau, E. (2013b), « About the illustrative use of the aller + infinitive periphrasis in French », in Labeau, E. et Bres, J. (éd.), Evolution in Romance Verbal Systems, Bern et Berlin : Peter Lang, 171-202.

Bündgen, M. (1996), « Zur pragmatischen Bedeutung der deutschen und franzosischen Futur », Nouveaux Cahiers d'Allemand, $1: 61-78$.

Bybee, J., Pagliuca, W. et Perkins, R. (1991), « Back to the future », in Traugott, E.C. et Heine, B. (éd.), Approaches to grammaticalization, vol. II, Amsterdam: Benjamins, 17-58.

Bybee, J., Perkins, R. et Pagliuca, W. (1994), The Evolution of grammar. Tense, aspect, modality in the languages of the world, Chicago, Londres : the University of Chicago Press.

Confais, J.P. (1992), « No future? Les 'Futurs' du français et de l'allemand », in Gréciano, G. et Kleiber, G. (éd.), Systèmes interactifs. Mélanges en l'honneur de Jean David (Recherches Linguistiques XVI), Paris : Klincksieck, 81-91.

Confais, J.P. (1995), Temps-mode-aspect : les approches des morphèmes verbaux et leurs problèmes à l'exemple du français et de l'allemand, $2^{\mathrm{e}}$ éd. revue et augmentée, Toulouse : Presses universitaires du Mirail.

Dahl, O. (1985), Tense and Aspect Systems, Oxford: Blackwell. 
Dahl, O. (1996), « Das Tempussystem des Deutschen im typologischen Vergleich », in Lang, E. et Zifonun, G. (éds), Deutsch - typologisch (Jahrbuch IDS 1995), Berlin, New York: Mouton de Gruyter, 359-368.

Dahl, O. (2000), « The Grammar of future time reference in European languages », in Dahl, O. (éd.), Tense and aspect in the languages of Europe (Eurotyp 6), Berlin, New York : Mouton de Gruyter, 309-328.

Damourette, J. et Pichon, E. (1911-1936), Des mots à la pensée. Essai de Grammaire de la Langue Française, Tome 5, Paris : d'Artrey.

Dendale, P. (2001), « Le futur conjectural versus devoir épistémique : différences de valeur et restrictions d'emploi », Le Français moderne, 69, $1: 1-20$.

Fleischman, S. (1982), The Future in thought and language - Diachronic evidence from Romance, Cambridge : Cambridge University Press.

Lansari, L. (2010), « ‘On va dire' : modalisation du dire et dénomination », in Frath, P., Lansari, L. et Pauchard, J. (éd.), Res per nomen II - Langue, référence et anthropologie, Reims : Epure, 277-295.

Larreya, P. (2008), « Autour du futur », in Pauchard, J. (éd.), Auxiliaire et auxiliation/ expression de la futurité, Reims : Epure, 131-143.

Martin, R. (1987), Langage et croyance, Bruxelles : Mardaga.

Rebotier, A. (2005), « Le Présent futural en allemand et en français », in Schmitt, C. et Wotjak, B. (éds.), Beitrage zum romanisch-deutschen und innerromanischen Sprachvergleich, Akten der gleichnamigen internationalen Arbeitstagung (Leipzig, 4. 10. - 6.10. 2003), vol. 2, Bonn : Romanischer Verlag, 267-278.

Rebotier, A. (2006), « Le Futur de l'allemand », Corela [en ligne], 4, 1. Disponible sur : http:// edel.univ-poitiers.fr/corela/document.php?id=1172

Rebotier, A. (2009), « Le Futur de l'allemand en comparaison avec les langues romanes : esquisse d'une définition d'une catégorie translinguistique de Futur », Faits de Langue 33, 69-78.

Rebotier, A. (2010), « Temps verbaux et référence temporelle : l'exemple des énoncés référant à l'avenir en français, en italien et en allemand », in Frath, P., Lansari, L. et Pauchard, J. (éd.), Res per nomen II - Langue, référence et anthropologie, 403420.

Rebotier, A. (2014), « Métaphores temporelles : les emplois non temporels des outils linguistiques de la temporalité », in Hilgert, E., Palma, S., Frath, P. et Daval, R., Les théories du sens et de la référence. Hommage à Georges Kleiber, Reims : EPURe, 341-358.

Schrott, A. (1997), Futuritat im Franzosischen der Gegenwart. Semantik und Pragmatik der Tempora der Zukunft, Romanica Monacensia 50, Tübingen : Narr.

Traugott, E.C. et Konig, E. (1991), « The Semantics-Pragmatics of Grammaticalization Revisited », in Traugott, E.C. \& Heine, B., Approaches to grammaticalization, Amsterdam : Benjamins, 189-218.

Vet, C. (1993), « Conditions d'emploi et interprétation des temps futurs du français », Verbum, 16, 4, 71-84.

Vetters, C. (1996), Temps, aspect et narration, Amsterdam, Atlanta : Rodopi.

Vetters, C. et Lières, A. (2009). «Quand une périphrase devient temps verbal : le cas d'aller + infinitif », Faits de Langue, 33, 27-36. 
Weber, H. (1954), Das Tempussystem des Deutschen und des Franzosischen. Übersetzungs- und Strukturprobleme, Bern : A. Francke.

Wilmet, M. (2003), Grammaire critique du français, $3^{e}$ édition, Bruxelles : Duculot.

\section{CORPUS}

Brecht, B. (1964), Der gute Mensch von Sezuan, Frankfurt/Main : Suhrkamp, 7-24.

Brecht, B. (1956), « La Bonne âme du Sé-Tchouan », traduit par J. Stem \& G. Serreau, in Théâtre complet V, Paris : L'Arche, 11-25.

Brecht, B. (1958), L'Anima buona del Sezuan, Turin : Einaudi, 13-27.

Fruttero, C., et Lucentini, F. (1979), A che punto è la notte, Milan, Mondadori, 7-102. (F\&L)

Fruttero, C., et Lucentini, F. (1981), Wie weit ist die Nacht, Munich : Piper, 7-97.

Fruttero, C., et Lucentini, F. (1981), La Nuit du grandBoss, traduit par J-C. Zancarini, Paris : Grasset, 11-150.

Pennac, D. (1997), Messieurs les enfants, Paris : Gallimard, 13-86.

Pennac, D. (1999), Grofie Kinder - kleine Eltern, traduit par E. Passet, Cologne : Kiepenheuer \& Witsch, 15-89.

Pennac, D. (2000), Signori bambini, traduit par Y. Melaouah, Milan : Feltrini, 11-67.

Pirandello, L., La Balia / Die Amme, traduit par M. Rossner, disponible sur : http:// www.pirandelloweb.com/

Pirandello, L. (1973), Nouvelles pour une année, II, traduit par H. Valot et G. Piroué, Paris : Gallimard, 449-472.

\section{NOTES}

1. Ce critère a été retenu pour sa clarté. Distinguer emplois temporels et emplois modaux est plus délicat, parce que les frontières de la catégorie " emplois modaux », qui regroupe des phénomènes très divers, sont plus difficiles à établir. Il est en particulier difficile de ne pas trouver de valeur modale dans tous les énoncés référant à l'avenir. D'autre part, certains emplois comme le futur historique ne sont ni temporels ni modaux. Enfin, si l'on admet que les emplois modaux et logiques sont issus des emplois temporels, les valeurs modales apparaissent dans un premier temps comme des interprétations pragmatiques ou des effets de sens (Traugott \& Konig 1991, 194). La disparition du sens temporel est la garantie que la forme a véritablement changé de sens et que la valeur modale lui appartient, en ce sens les énoncés non futuraux permettent mieux de définir ces nouvelles valeurs. Le choix d'un critère purement temporel a pour conséquence que certains des énoncés futuraux étudiés sous 3. ont des valeurs modales caractérisées, comme l'allure extraordinaire. Ces valeurs pourront être identifiées et mises en relation avec les catégories issues de la comparaison des langues en aveugle.

2. S'il est impossible d'affecter des critères formels donnés à un stade de grammaticalisation précis, il semble bien qu'il y ait corrélation entre l'évolution formelle et l'évolution sémantique (Bybee, Pagliuca \& Perkins 1991, 39 ; Dahl 1996, 362) : grammaticalisation sémantique et grammaticalisation formelle sont deux processus non synchronisés, mais parallèles. 
3. Pour Vet, tous les FP sans complément de temps, comme les exemples (5) à (7), sont des emplois de phase. Un argument en faveur d'une lecture temporelle de ces exemples, ou du moins indifférenciée entre phase et temps, est la possibilité d'interroger en réponse sur la date du procès, ce qui n'est pas possible avec une expression purement de phase comme être sur le point de :

Paul va se marier. - Quand? / Dans combien de temps?

Paul est sur le point de se marier. *Quand?

En raison de cette ambiguïté, je ne tenterai pas, dans la partie 3 ci-dessous, de classer les occurrences de FP systématiquement en emplois temporels et emplois de phase.

4. Si les verbes modaux font partie des sources lexicales des futurs, la modalité d'énoncé (modalité épistémique, particules illocutoires) est souvent citée comme une étape de grammaticalisation ultérieure à la temporalité en général (Traugott \& Konig 1991, 189, Rebotier 2014).

5. Est exclu notamment le futur injonctif, dont la valeur d'ordre, comme le notent Barcelo (2007: 49) et Vet (1994), s'explique pragmatiquement, à partir de la valeur temporelle, et qui est d'ailleurs possible avec le FP comme avec le FS. L'expression de la phase, première étape dans la grammaticalisation, n'est pas non plus concernée, puisqu'elle situe bien le procès dans l'avenir, même si c'est indirectement.

6. C'est-à-dire non temporels. Il existe également des emplois futuraux de l'extraordinaire, cf. cidessous 3.2.1.

7. Toutes les traductions sont tirées du corpus (référence en fin d'article).

8. http://www.vexilla-regis.com/textevr/VOLKOPFPRTKRRASAINTEJEANNE\%20d.htm, consulté en juin 2014.

9. Le principe du corpus parallèle trilingue permet difficilement d'étudier l'oral (sauf en contexte de travail formel peu représentatif) et la langue écrite informelle, qui permettrait de voir les emplois les plus récents. Cependant, le corpus littéraire comporte des passages imitant l'oral (Pennac, Brecht).

10. FPF $: 2$ occurrences, PFF $: 2$, PPF $: 1$, PFP $: 5$. Le corpus (343 occurrences) comporte en outre des énoncés qui n'entrent pas dans ces huit catégories (traduction dans au moins une langue soit par une forme verbale qui n'est ni un présent, ni un futur, soit sans verbe correspondant, soit avec référence temporelle non futurale) mais qui sont pris en compte dans les calculs concernant uniquement les langues où les critères (temps présent ou futur, référence future) sont remplis.

11. L'expression de l'intention n'est pas limitée à la première personne. Ont été retenus comme dépendants de la volonté tous les procès dont le sujet grammatical est agent.

12. Ce qui laisse penser qu'être en train, qui correspond mieux à stare per et im Begriff sein, n'est pas synonyme du FP, même dans ses emplois de phase.

13. La présence de noch dans (31") et (32") contribue au sens futural.

14. Pour cette raison (sens présentai en italien), l'exemple 31 n'est pas compté dans les catégories du schéma 2. 


\section{RÉSUMÉS}

Le futur périphrastique français avec aller : un renvoi spécifique à l'avenir ou un temps en voie de grammaticalisation? - Une approche contrastive.

Le futur périphrastique français (FP) semble être un temps en voie de grammaticalisation : forme composée, plus récente que le futur simple (FS), il peut focaliser la phase préparatoire du procès à venir, et même dans ses emplois de temps, il est souvent décrit comme porteur d'un point de vue présent sur le procès. On peut se demander si ses spécificités par opposition au FS sont dues à sa grammaticalisation inachevée ou s'il suit un autre chemin de grammaticalisation conforme au sens de son auxiliaire aller. Cet article cherche à répondre en dressant le tableau de ses emplois actuels, temporels et non temporels.

Contrairement à ce qui est souvent affirmé, le FP est apte à tous les emplois modaux non temporels du FS (générique, épistémique, performatif, narratif) et n'a développé aucun emploi modal spécifique, l'extraordinaire pouvant être considéré comme une forme homonyme. Les emplois temporels sont évalués par rapport à une catégorie translinguistique de futur établie sur la base d'un corpus parallèle français, italien et allemand, qui permet de distinguer des emplois prototypiques et des emplois marginaux. Le FP apparaît de façon privilégiée dans les zones marginales, où le renvoi à l'avenir est indirect, mais il n'est pas absent des emplois typiques, où le FS domine. Le tableau est donc bien celui d'une forme en train de conquérir les emplois du FS. Toutefois, l'éloignement temporel est également un critère pertinent dans la concurrence entre FP et FS et indiquerait une spécialisation des deux formes.

The French periphrastic future (PF) with aller (go) seems to be still in the process of grammaticalization into a tense. The PF represents a compound form and it is more recent than the simple future (SF). It can be used as a phase expression, focusing on the phase before the future process, and even when used as a tense, it is often said to show the future process as viewed from the present. One may ask whether its specific features are due to an unfinished grammaticalization or whether it is following another path of grammaticalization according to the nature of its auxiliary. This paper aims to answer this precise question, by providing a picture of its current temporal as well as non-temporal uses. Contrary to what is often said, the PF is suited to all non-temporal modal uses of the SF (generic, epistemic, performative and narrative) and has developed no particular modal use, since the mirative use can be considered a homonymous form. The temporal uses are assessed in relation to a cross-linguistic category of future tense, defined on the basis of a French-German-Italian parallel corpus, making it possible to distinguish between prototypical uses of a future tense and marginal ones. The PF tends to appear in the marginal zones, with an indirect reference to the future, but is nevertheless apparent in the typical uses, where the SF prevails. Thus, the picture shows an emerging form conquering the uses of the SF. However, temporal remoteness is also a relevant factor in the competition between SF and PF and may indicate a specialization of each form.

\section{INDEX}

Index géographique : France

Mots-clés : futur, aller, futur proche, futur périphrastique, grammaticalisation, français, temps, allemand, italien, modalité 
AUTEUR

AUDE REBOTIER

CeliSo (Paris IV), CIRLEP (Reims), Université de Reims Champagne-Ardenne 